\title{
Complex moments of parton distributions and new sum rules
}

\section{Citation}

Bourrely, C., J. Soffer, and Tai Tsun Wu. 1987. “Complex Moments of Parton Distributions and New Sum Rules." Physical Review Letters 59 (18): 2009-11. https://doi.org/10.1103/ physrevlett.59.2009.

\section{Permanent link}

http://nrs.harvard.edu/urn-3:HUL.InstRepos:41555828

\section{Terms of Use}

This article was downloaded from Harvard University's DASH repository, and is made available under the terms and conditions applicable to Other Posted Material, as set forth at http:// nrs.harvard.edu/urn-3:HUL.InstRepos:dash.current.terms-of-use\#LAA

\section{Share Your Story}

The Harvard community has made this article openly available.

Please share how this access benefits you. Submit a story.

Accessibility 


\title{
Complex Moments of Parton Distributions and New Sum Rules
}

\author{
C. Bourrely and J. Soffer \\ Centre de Physique Théorique du Centre National de la Recherche Scientifique, Faculté des Sciences Luminy, \\ 13288 Marseille Cédex 9, France \\ and
}

Tai Tsun Wu

CERN, Geneva, Switzerland, and

Gordon McKay Laboratory, Harvard University, Cambridge, Massachusetts 02138

(Received 25 June 1987)

\begin{abstract}
The $Q^{2}$ evolution of quark and gluon distributions predicted by perturbative QCD is reexamined by consideration of the complex moments of these distributions. This analytic continuation leads us to derive several interesting new sum rules which depend on the number of flavors.
\end{abstract}

PACS numbers: $12.38 . \mathrm{Bx}$

The predictions of perturbative QCD for the evolution of quark and gluon structure functions inside a nucleon have been extensively studied. They play an essential role in the discussion of many standard-model and newphysics issues, in particular for the evaluation of production rates at present and future hadron colliders. ${ }^{1}$ Fundamental interactions involve the collisions of quarks and gluons whose distributions $f_{i}\left(x, Q^{2}\right)$ are extracted from deep-inelastic data at low $Q^{2}$ and must be extrapolated at very high $Q^{2}$ with use of the Altarelli-Parisi equations. $^{2}$ In the determination of these functions for the unpolarized case as well as for the polarized case, various sum rules provide important constraints by means of the existence of conserved quantities, that is $Q^{2}$ independent (e.g., momentum conservation allowing the normalization of the gluon distribution not directly accessible in electroproduction) which have been very useful. These sum rules might also be used to check the very-small- $x$ behavior, a region which is crucial at supercollider energies but poorly known at the moment because of the lack of data. ${ }^{3}$ We will have to wait for HERA to explore the structure functions in a wider kinematic region including very-small- $x$ values.

In this Letter we propose to consider the complex moments of the quark and gluon polarized distributions which lead us to the discovery of a continuum of new sum rules. For a given parton (quark or gluon) let us denote by $f_{ \pm}\left(x, Q^{2}\right)$ the parton distributions in a polarized nucleon, either with helicity parallel $(+)$ or anti- parallel $(-)$ to the nucleon helicity. We will be particularly interested in the quark and gluon helicity asymmetries $\Delta q=q_{+}-q_{-}$and $\Delta G=G_{+}-G_{-}$. The singlet quark asymmetry, defined as $\Delta \Sigma=\Sigma_{f}\left(\Delta q_{f}+\Delta \bar{q}_{f}\right)$, involves a summation over all quark flavors and the corresponding antiquark contributions $\Delta \bar{q}_{f}$. For any distribution $f(x, t)$ where $t=\ln \left(Q^{2} / \Lambda^{2}\right)$, the $n$th moment is defined as

$$
f_{n}(t)=\int_{0}^{1} d x x^{n-1} f(x, t)
$$

$\Delta \Sigma_{n}$ and $\Delta G_{n}$ are coupled by the evolution equations abbreviated as ${ }^{4}$

$$
\frac{d}{d t}\left(\begin{array}{c}
\Delta \Sigma_{n} \\
\Delta G_{n}
\end{array}\right)=\frac{\alpha(t)}{2 \pi}\left(\begin{array}{ll}
\alpha_{n} & \beta_{n} \\
\gamma_{n} & \delta_{n}
\end{array}\right) \otimes\left(\begin{array}{l}
\Delta \Sigma_{n} \\
\Delta G_{n}
\end{array}\right),
$$

where $\alpha(t)=1 / b t$ is the strong coupling constant and $b=\left(33-2 N_{f}\right) / 12 \pi$. These equations have been usually considered for $n$ positive integer, and we have

$$
\begin{aligned}
& \alpha_{n}=\frac{4}{3}\left(\frac{3}{2}-\frac{1}{n}-\frac{1}{n+1}-2 \sum_{k=1}^{n-1} \frac{1}{k}\right), \\
& \beta_{n}=N_{f}\left(-\frac{1}{n}+\frac{2}{n+1}\right), \quad \gamma_{n}=\frac{4}{3}\left(\frac{2}{n}-\frac{1}{n+1}\right), \\
& \delta_{n}=3\left(\frac{11}{6}-\frac{N_{f}}{9}+\frac{2}{n}-\frac{4}{n+1}-2 \sum_{k=1}^{n-1} \frac{1}{k}\right),
\end{aligned}
$$

where $N_{f}$ is the number of flavors.

The solutions may be written as ${ }^{4}$

$$
\left(\begin{array}{c}
\Delta \Sigma_{n} \\
\Delta G_{n}
\end{array}\right)=\left(\Delta a_{n}^{+}-\Delta a_{n}^{-}\right)^{-1}\left[\left(\Delta \Sigma_{n_{0}}+\Delta a_{n}^{+} \Delta G_{n_{0}}\right)\left(\begin{array}{c}
-\Delta a_{n}^{-} \\
1
\end{array}\right)\left(\frac{t}{t_{0}}\right)^{\Delta d_{n}^{+}}+\left(\Delta \Sigma_{n_{0}}+\Delta a_{n}^{-} \Delta G_{n_{0}}\right)\left(\begin{array}{c}
\Delta a_{n}^{+} \\
-1
\end{array}\right)\left(\frac{t}{t_{0}}\right)^{\Delta d_{n}^{-}}\right],
$$

where

$$
\Delta a_{n}^{ \pm}=\left(\delta_{n}-\alpha_{n} \pm \Delta_{n}\right) / 2 \gamma_{n}, \quad \Delta_{n}=\left[\left(\alpha_{n}-\delta_{n}\right)^{2}+4 \beta_{n} \gamma_{n}\right]^{1 / 2},
$$

and $\Delta d_{n}^{ \pm}=(4 \pi b)^{-1}\left(\alpha_{n}+\delta_{n} \pm \Delta_{n}\right)$ are the eigenvalues of the $2 \times 2$ matrix in Eq. (2). Clearly $\Delta \Sigma_{n_{0}}=\Delta \Sigma_{n}\left(t=t_{0}\right)$ and 
$\Delta G_{n_{0}}=\Delta G_{n}\left(t=t_{0}\right)$ are the input values. The generalization to noninteger values can be obtained if one replaces the discrete sum occurring in $\alpha_{n}$ and $\delta_{n}$ by an analytic function of $n$. This can be done since

$$
\sum_{k=1}^{n-1} \frac{1}{k}=\psi(n)+\gamma,
$$

where $\psi(n)$ is the well-known digamma function, and $\gamma=0.577$ is the Euler constant. It was used for the first time by Einhorn and Soffer, ${ }^{5}$ where it was noted that the vanishing of the dominant eigenvalue $\Delta d_{v}^{+}=0$ for $1<v<2$ leads to a conserved quantity, namely the corresponding eigenvector:

$$
u_{v}^{+}=\Delta \Sigma_{v}+\Delta a_{v}^{+} \Delta G_{v}
$$

is independent of $Q^{2}$ with $0<\Delta a_{v}^{+} \leq 1$. The exact values of $v$ and $\Delta a_{v}^{+}$vary with the number of flavors and decrease slightly when $N_{f}$ increases from $N_{f}=3$ to $N_{f}=6$. This can be further generalized by considering complex $n$ values. Here we have a situation analogous to that encountered by Regge, ${ }^{6}$ who made the first attempt to investigate the complex $l$ plane starting from the partial-wave expansion of a scattering amplitude given by $f(s, t)=\sum_{l}(2 l+1) a_{l}(s) P_{l}$ and defined for integer $l$ values.

Our purpose is then to study the condition

$$
\operatorname{Re}\left(\Delta d_{n}^{+}\right)=0
$$

for $n=n_{1}+i n_{2}$. This condition determines the existence of trajectories in the complex $n$ plane, and along these trajectories the modulus of the complex number

$$
u_{n}^{+}=\Delta \Sigma_{n}+\Delta a_{n}^{+} \Delta G_{n}
$$

is independent of $Q^{2}$. Clearly $F(n) \equiv \Delta d_{n}^{+}$is a real analytic function of $n$, so if $n$ is a solution of Eq. (7), $n^{*}$ the complex conjugate of $n$ is also a solution. The trajectories are symmetric around the axis $n_{2}=0$, and we will show them only in the upper half plane. If one writes the condition $\operatorname{Re} F(n)=0$ in the explicit form $n_{2}=f\left(n_{1}\right)$, the derivative $d f / d n_{1}$ can be simply expressed in terms of

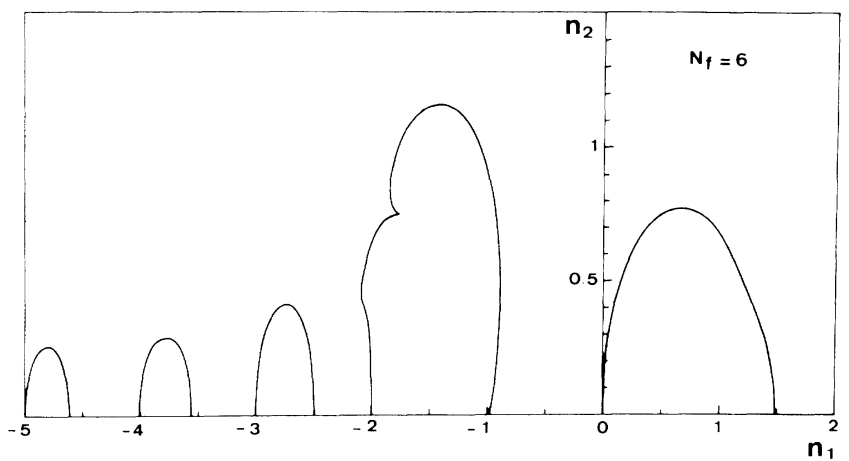

FIG. 1. Trajectories determined by the solutions of Eq. (7) for $N_{f}=6$ in the upper half plane. $d F / d n$ because we have

$$
\frac{d f}{d n_{1}}=\frac{\operatorname{Re}(d F / d n)}{\operatorname{Im}(d F / d n)}
$$

which is very useful for an accurate determination of the trajectories. In Fig. 1 we show these trajectories for $N_{f}=6$, and in Fig. 2 we give some details in the interesting region between $n_{1}=-1$ and $n_{1}=-2$ for two values of $N_{f}$. Near $n_{1}=-1$, the trajectory has two inflection points for $N_{f}=3$ which have disappeared for $N_{f}=6$. In Fig. 3 we give the details of the special structure occurring around $n_{1}=-1.7$. A careful study of the derivative shows that it is not a cusp but a point where two straight lines intersect with a certain angle. This angle becomes smaller when $N_{f}$ increases, and the angular point disappears for $N_{f}>6$. This suggests that $N_{f}=6$ corresponds perhaps to a critical value and more work remains to be done. There is a formal analogy between these trajectories and the Regge trajectories corresponding to the pole of $a_{l}(s)$ in the complex $l$ plane, whose real part $\alpha(t)$ gives $\left(s / s_{0}\right)^{\alpha(t)}$ for the high-energy behavior of $f(s, t)$.

Clearly there are more sum rules. In particular if $u_{n}^{+}$ denotes the eigenvector corresponding to $\Delta d_{n}^{+}$, for any couple of complex numbers $n$ and $n^{\prime}$ such that

$$
\operatorname{Re}\left(\Delta d_{n}^{+}+\Delta d_{n^{\prime}}^{+}\right)=0,
$$

the quantity $u_{n}^{+} u_{n^{\prime}}^{+}$is independent of $Q^{2}$, and this can be further generalized.

The usefulness of these sum rules can be illustrated by our considering a testing set of polarized structure functions which are poorly known because of the scarcity of

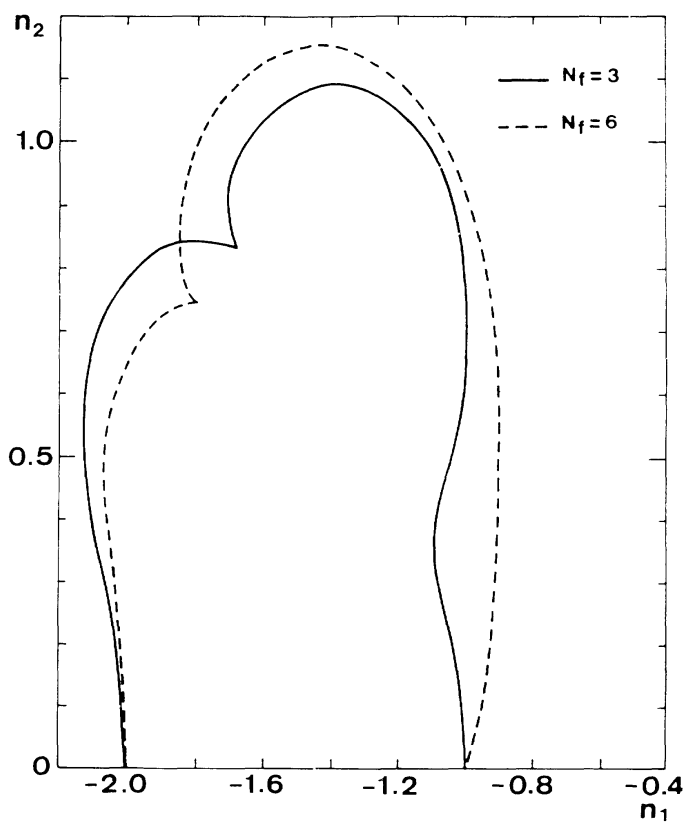

FIG. 2. A comparison of the trajectories for $N_{f}=3$ and $N_{f}=6$ in a limited region of the complex $n$ plane. 


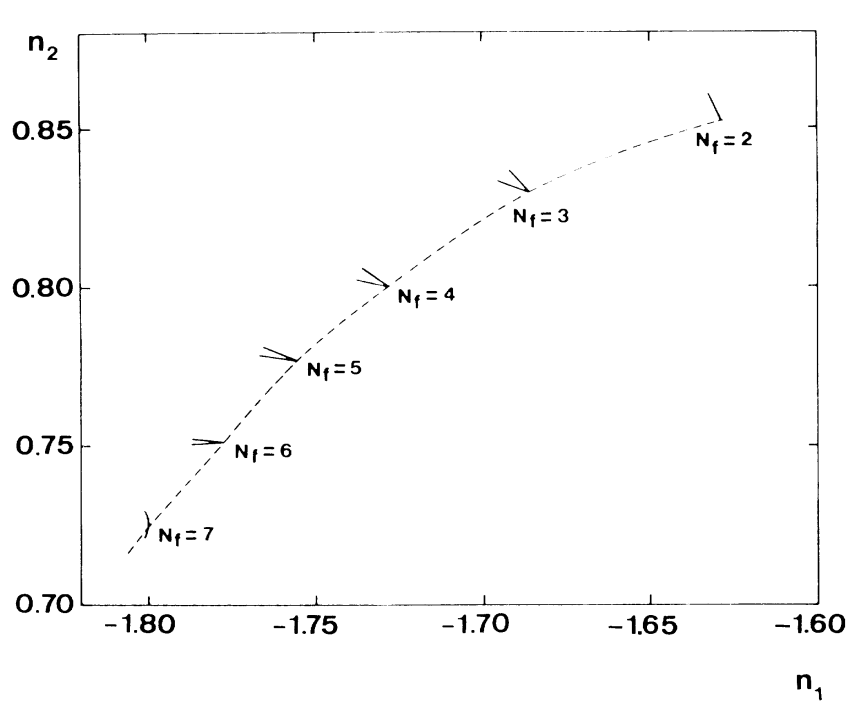

FIG. 3. Evolution of a special structure of the trajectory for different values of $N_{f}$.
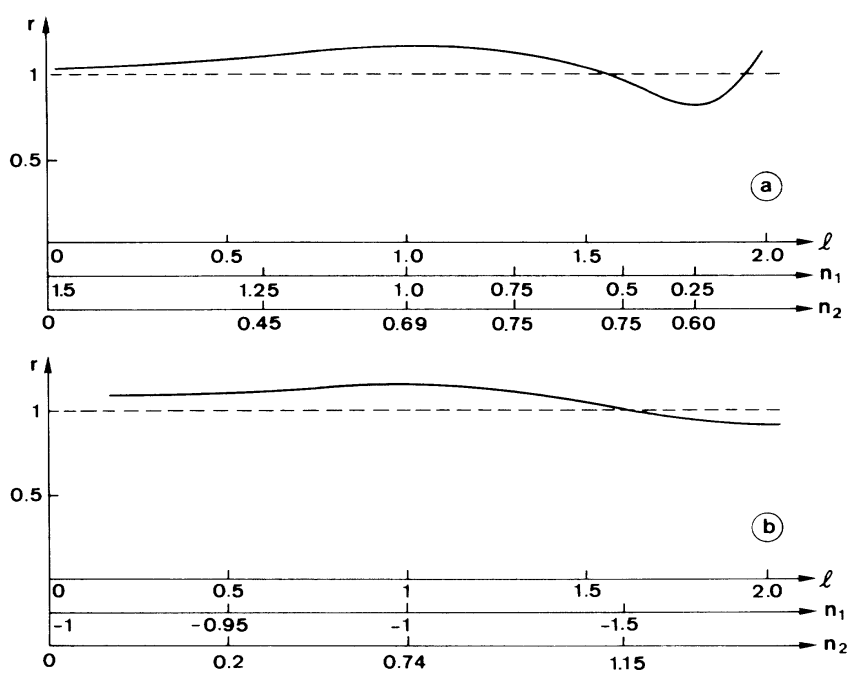

FIG. 4. Ratio $r$ vs $l$, the length along the trajectory (a) for the right-hand-side branch $\left(n_{1}>0\right)$, (b) for the first lefthand-side branch.

data. As an approximation, let us take at high $Q^{2}$ for $N_{f}=6$

$$
\begin{aligned}
& \Delta \Sigma(x, S)=\sqrt{S} \exp \left\{\left[1.23(\ln l / x)^{1 / 2}-1.5 \sqrt{S}\right] \sqrt{S}\right\} x-0.35(1-x)^{3}, \\
& \Delta G(x, S)=\exp \left\{\left[3.4(\ln l / x)^{1 / 2}-4.5 \sqrt{S}\right] \sqrt{S}\right\}(1-x)^{6},
\end{aligned}
$$

where $S=\ln t / t_{0}$. We have then calculated $\left|u_{n}^{+}\right|$along the trajectories for different values of $Q^{2}$. Due to the expressions for $\Delta \Sigma$ and $\Delta G$ this can be done analytically for any complex moment in terms of a sum of error functions. We have plotted in Fig. 4 the ratio $r$ of $\left|u_{n}^{+}\right|$for $Q^{2}=10^{8} \mathrm{GeV}^{2}$ to $\left|u_{n}^{+}\right|$for $Q^{2}=10^{5} \mathrm{GeV}^{2}$ versus the length $l$ along two branches of the trajectories starting on the intercept on the right; we also indicate some corresponding values of $n_{1}$ and $n_{2}$ taken from Fig. 1. Although along the trajectories the value of $\left|u_{n}^{+}\right|$varies substantially, we observe that $r$ remains close to one within at most $20 \%$ which allows us to evaluate the quality of the approximation made in Eq. (11).

The consideration of complex moments for the parton distributions and the existence of trajectories in the complex $n$ plane giving a continuum of sum rules is undoubtedly a new concept. It has been illustrated in a special case, and it certainly deserves a more detailed investigation, in particular for the unpolarized distributions which are accurately known from existing data.
One of us (T.T.W.) acknowledges support in part by the U.S. Department of Energy under Grant No. DEFG02-84 ER 40158. Centre de Physique Théorique is Laboratoire propre No. LP7061 du Centre National de la Recherche Scientifique.

${ }^{1}$ E. Eichten, I. Hinchliffe, K. Lane, and C. Quigg, Rev. Mod. Phys. 56, 579 (1984).

${ }^{2}$ G. Altarelli and G. Parisi, Nucl. Phys. B126, 298 (1977).

${ }^{3}$ For a recent review of the subject, see J. Feltesse, in Proceedings of the International Europhysics Conference on High Energy Physics, Bari, Italy, 1985, edited by L. Nitti and G. Preparata (European Physical Society, Geneva, 1985), p. 979.

${ }^{4}$ G. Altarelli, Phys. Rep. 81, 1 (1982).

${ }^{5}$ M. Einhorn and J. Soffer, Nucl. Phys. B274, 714 (1986).

${ }^{6}$ T. Regge, Nuovo Cimento 14, 951 (1959), and 18, 947 (1960). 